\title{
Programa Nacional de Vacinação 2017 e vacinação antitetânica
}

Fabrizio Cossutta*

\section{RESUMO}

O tétano é uma doença infeciosa grave provocada pela contaminação de feridas por esporos de Clostridium tetani. A vacinação é a única intervenção capaz de produzir imunidade, sendo necessária a administração de reforços para manter níveis de anticorpos protetores e duradouros. Recentemente foram apresentadas alterações ao atual esquema de vacinação português, a entrar em vigor a partir de 2017. Estas alterações reduzem o número de reforços aconselhados, de acordo com as recomendações de outros países da União Europeia. O objetivo deste artigo é fundamentar e analisar a evidência científica que sustenta estas alterações.

Palavras-chave: Tétano; Vacina antitetânica; Programas de vacinação

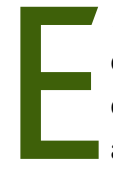
m junho de 2016, a Direção-Geral da Saúde (DGS) divulgou algumas alterações previstas para o esquema de vacinação português, a entrar em vigor a partir de janeiro de 2017.

Entre as várias alterações destaca-se a alteração da periodicidade dos reforços da vacina antitetânica, com consequente redução de reforços administrados em idade adulta.

O objetivo deste artigo é fundamentar e analisar as motivações que sustentam estas alterações.

\section{A DOENÇA}

O tétano é uma doença grave, potencialmente fatal, que ocorre após contaminação de qualquer tipo de ferida com esporos da bactéria anaeróbia Clostridium tetani. Os esporos do C.tetani são ubíquos no solo, particularmente em áreas de clima quente e húmido, podendo ser disseminados a partir do intestino de vários animais, entre os quais os cavalos.

Após inoculação, os esporos podem transformar-se em bacilos produtores da tetanospasmina, a neurotoxina causadora da doença tetânica.

O período de incubação varia geralmente entre três a 21 dias (mediana sete dias, intervalo 0-60). A apresentação

*UCSP Penha de França, ACES Lisboa Central clássica, presente em cerca de $80 \%$ dos casos, é caracterizada por um quadro inicial de espasmo dos músculos faciais (trismus ou risus sardonicus), que progride para espasmo da musculatura dorsal (opistotonus) e, finalmente, espasmo muscular generalizado (tetanoespasmo).

Nos extremos etários, a mortalidade ronda os $100 \%$, baixando até $10-20 \%$ nos casos atendidos em hospitais com cuidados adequados. ${ }^{1}$

Os casos de tétano neonatal ocorrem em recém-nascidos de mãe não imunizada após um parto com falta de assepsia (higiene do local e uso de ferramentas não esterilizadas), por contaminação direta do coto umbilical. ${ }^{2}$

Aimunidade é mediada por anticorpos e depende da capacidade destas antitoxinas de neutralizar a tetanospasmina. Não existe imunidade natural contra o tétano, sendo somente obtida a partir de imunização ativa (vacinação) ou passiva (administração de imunoglobulina). Somente através da administração de várias doses de vacina antitetânica é possível atingir uma imunidade considerada duradora. Contudo, por ser uma doença infeciosa, mas não contagiosa, a vacinação não produz imunidade de grupo.

\section{A SITUAÇÃO EM PORTUGAL}

Em Portugal, a vacina antitetânica foi introduzida com caráter obrigatório pelo Decreto-Lei n. ${ }^{\circ} 44.198$, de 20 de fevereiro de 1962. Contudo, a aplicação deste decreto concretizou-se só em 1965 com a introdução desta vacina no 
primeiro Programa Nacional de Vacinação (PNV), assegurando uma ampla cobertura populacional através da distribuição universal e gratuita de vacinas à população, de acordo com um calendário definido e seguindo as orientações técnicas estabelecidas.

Esta medida proporcionou uma diminuição marcada da morbilidade associada ao tétano (Quadro I) e, juntamente com a melhoria das condições de higiene, levou a que o último caso de tétano neonatal ocorresse em 1997.

Apesar do grande avanço alcançado com a vacinação universal, em Portugal, como na Europa, continuam a registar-se casos de tétano, sobretudo em idosos do sexo feminino não vacinados ou com vacinação incompleta.

Relativamente a Portugal, de acordo com o trabalho realizado por Castro e colaboradores, ${ }^{3}$ na década de 19932002 ocorreram 210 casos de tétano na região norte do País, dos quais a maioria em indivíduos acima de 65 anos (73\% do sexo feminino).

No triénio 2000-2002 e na mesma região foram comunicados 17 casos (dois óbitos), todos na sequência de internamento hospitalar, sendo a maioria em adultos idosos não corretamente vacinados. ${ }^{3}$

Os últimos dados disponíveis da DGS revelam que na década de 2006-15 foram declarados 33 casos em Portugal, que resultaram em 15 óbitos. ${ }^{4}$

No que diz respeito à vacinação antitetânica, o PNV português em vigor até 2016 previa a administração de seis doses desde o nascimento até à adolescência (dois, quatro, seis, 18 meses, cinco/seis e 10/13 anos), com reforços de 10 em 10 anos durante toda a vida com vacina combinada do tipo «Td» (vacina dupla, contra o tétano e com dose reduzida contra a difteria).

No que diz respeito ao cumprimento deste, os dados divulgados pela DGS relativos a dezembro de 2012 mostravam uma taxa de cobertura muito elevada na infância (>95\%), mas apenas $60 \%$ da coorte que completou 65 anos de idade se encontrava imunizada contra o tétano (indivíduos nascidos em 1947). ${ }^{2}$ Estes dados, juntamente com as características demográficas dos casos de tétano da última década, reforçam a necessidade de incentivar a vacina antitetânica na população adulta e, sobretudo, idosa.

Ao mesmo tempo, vários autores têm questionado a necessidade da utilização de reforços a cada 10 anos, ${ }^{5-12} \mathrm{com}$ autores portugueses a sugerirem a possibilidade de espaçar os reforços em idade adulta a cada $20 \operatorname{anos}^{13}$ e outros a sugerirem um reforço único aos 65 anos, com base em dados de custo-eficácia. ${ }^{14}$ É importante relembrar que a utilização

\begin{tabular}{|c|c|c|c|}
\hline \multicolumn{4}{|c|}{$\begin{array}{l}\text { QUADRO I. Casos notificados de tétano, óbitos e taxa de } \\
\text { mortalidade por década }\end{array}$} \\
\hline Década & Casos notificados & Óbitos & Taxa de mortalidade \\
\hline $1956-65$ & 3.923 & 2.625 & $67 \%$ \\
\hline 2002-11 & 55 & 10 & $18 \%$ \\
\hline $2006-15$ & 33 & 15 & $45 \%$ \\
\hline
\end{tabular}

Fonte: Direção-Geral da Saúde. ${ }^{4}$

de reforços a cada 10 anos aumenta a despesa em saúde e o risco de reações adversas que, apesar de raramente graves, são diretamente proporcionais aos níveis de anticorpos prévios e, consequentemente, ao número de reforços já recebidos. ${ }^{15}$ As reações adversas com evidência científica a suportar a relação de causalidade são a neuropatia do plexo braquial (0,5 a um caso em cada 100.000 vacinas administradas) e a reação anafilática (1,6 casos por milhão) ${ }^{6}$

Relativamente à síndroma de Guillan-Barré, apesar de ter sido descrita após administração da vacina combinada «Td», ${ }^{16}$ estudos recentes e mais robustos não confirmaram essa associação. ${ }^{17}$

É importante realçar que, tendo em conta o volume de vacinas administradas todos os anos, as reações adversas acima descritas são mais frequentes que a doença alvo da vacinação. $^{6}$

Em junho de 2016 foi apresentado o novo PNV, a entrar em vigor a partir de janeiro de 2017. No que diz respeito à vacinação contra o tétano, a principal alteração está relacionada com os intervalos recomendados para a administração dos reforços (Quadro II).

É importante salientar que a partir dos 65 anos os reforços voltam a ser decenais. Esta recomendação baseiase no conceito da imunosenescência: segundo esta teoria, o sistema imunitário de indivíduos idosos terá menor capacidade para produzir uma resposta imune efetiva e duradora. ${ }^{4,18}$ Os dados publicados parecem confirmar esta hipótese quanto à vacinação antipneumocócica, contra a gripe e contra o herpes zoster, mas não relativamente à vacina do tétano, ${ }^{18-20}$ sendo assim necessários mais estudos para determinar a real necessidade destes reforços.

\section{OS ESQUEMAS VACINAIS FORA DE PORTUGAL}

No Quadro II estão resumidas as recomendações relativas à periodicidade dos reforços na população adulta e idosa em diferentes países da União Europeia, Estados 


\begin{tabular}{|c|c|c|c|}
\hline & $\begin{array}{l}\text { Doses até idade adulta } \\
\qquad(<18 \text { anos })\end{array}$ & $\begin{array}{l}\text { Periodicidade reforços } \\
\text { na idade adulta }\end{array}$ & $\begin{array}{c}\text { Periodicidade reforços na população } \\
\text { idosa (>65 anos) }\end{array}$ \\
\hline Espanha $^{21}$ & 6 & \multicolumn{2}{|c|}{ Reforço único aos 65 anos } \\
\hline EUA $^{22}$ & 6 & \multicolumn{2}{|r|}{ Cada 10 anos } \\
\hline França $^{23}$ & 5 & Cada 20 anos & Cada 10 anos \\
\hline OMS $^{1}$ & 5 & \multicolumn{2}{|c|}{ Reforço único (serviço militar obrigatório ou gravidez) } \\
\hline Portugal (até 2016) & 6 & \multicolumn{2}{|r|}{ Cada 10 anos } \\
\hline Portugal 2017 & 6 & Cada 20 anos & Cada 10 anos \\
\hline Reino Unido ${ }^{24}$ & 5 & \multicolumn{2}{|c|}{ Reforço único para viajantes (avaliação caso a caso) } \\
\hline Suécia ${ }^{23}$ & 5 & \multicolumn{2}{|c|}{ Cada 20 anos } \\
\hline
\end{tabular}

Unidos da América (EUA) e as recomendações da Organização Mundial da Saúde (OMS).

No Reino Unido, por exemplo, está recomendada a primovacinação (dois, três e quatro meses), seguida de um primeiro reforço aos três/quatro anos (4-in-1 pre scholar booster) e de um segundo reforço aos 13/14 anos (3-in-1 teenage booster). É ainda aconselhada a administração de um terceiro reforço aos indivíduos adultos que viajam para zonas do mundo com sistemas de saúde deficitários. ${ }^{24}$

O recomendado pela OMS prevê a administração de seis doses no total - a primovacinação é seguida por dois reforços em idade escolar e um reforço em idade adulta (na altura da primeira gravidez na mulher e do serviço militar obrigatório no homem). Segundo os autores das recomendações mais recentes, este esquema produz anticorpos considerados protetores por um período de 20 a 30 anos. ${ }^{1}$

Relativamente a Espanha, o esquema de vacinação tem sofrido alterações recentes, impulsionadas também pelos trabalhos publicados por Luís Palomo ${ }^{12,25-27}$ desde 1987. No passado, o de vacinação era sobreponível ao português, com reforços na idade adulta a cada 10 anos também em indivíduos devidamente vacinados na infância e adolescência (seis doses). A partir de 2009, ${ }^{21}$ as novas recomendações aconselham a administração de um único reforço aos 65 anos para toda a população corretamente vacinada na infância, inclusive grávidas. Adicionalmente pode ser recomendado um reforço extra para adultos a viajar para zonas com sistemas de saúde deficitários. Relativamente aos adultos que receberam a primovacinação só na idade adulta é aconselhado administrar dois reforços decenais até perfazer um total de cinco doses.

\section{REFORÇOS COM VACINAÇÃO COMBINADA E RISCO DE DIFTERIA}

Historicamente, uma das principais críticas à alteração do esquema de reforço contra o tétano prende-se com o risco de reduzir os níveis de anticorpos contra a difteria.

A difteria é uma doença provocada pela toxina produzida por algumas bactérias anaeróbias da espécie Corynebacterium diphteriae. O único reservatório é o homem e a transmissão ocorre através do contacto do agente com as vias respiratórias superiores.

Contudo, é preciso ter em mente algumas considerações: ${ }^{6}$

1. A difteria sempre foi considerada uma doença pediátrica e a recomendação de administrar reforços contra a difteria na idade adulta só surgiu quando a vacina «Td» foi disponibilizada;

2. Nos países desenvolvidos, a difteria é uma doença do passado, sendo que o último caso documentado em Portugal ocorreu na década de 80;

3. A vacina «Td» confere imunidade duradoura contra o tétano mas não contra a difteria, pois os níveis de anticorpos contra a difteria diminuem mais rapidamente. Ainda assim, apesar de ser considerável a população adulta suscetível, não têm ocorrido casos;

4. A vacina contra a difteria desencadeia imunidade contra o toxoide diftérico e não contra a bactéria em si. Por esta razão, os indivíduos vacinados podem ainda ser portadores assintomáticos da bactéria C.diphteriae e poderão transmiti-la à população não vacinada;

5. Alguns autores têm sugerido a substituição dos reforços «Td» com reforços «Tdap», ${ }^{7}$ de forma a reduzir o risco de contágio entre adultos não imunes à tosse convulsa e recém-nascidos ainda não vacinados. Contudo, 


\begin{tabular}{|c|c|c|c|c|}
\hline \multirow[t]{2}{*}{ História imunológica } & \multicolumn{2}{|c|}{ Ferida de baixo risco } & \multicolumn{2}{|c|}{ Ferida de risco elevado } \\
\hline & Reforço & Imunoglobulina & Reforço & Imunoglobulina \\
\hline $\begin{array}{l}\text { Indeterminada ou } \\
<3 \text { doses }\end{array}$ & $\begin{array}{c}\text { Sim } \\
\text { (completar primo- } \\
\text {-vacinação, se necessário) }\end{array}$ & Não & $\begin{array}{c}\text { Sim } \\
\text { (completar primo- } \\
\text {-vacinação, se necessário) }\end{array}$ & Sim \\
\hline$\geq 3$ Doses & Não ${ }^{1}$ & Não & Não ${ }^{2}$ & Não ${ }^{3}$ \\
\hline \multicolumn{5}{|c|}{$\begin{array}{l}\text { Feridas ou queimaduras com extenso tecido desvitalizado, mordeduras, feridas punctiformes ou contaminadas com } \\
\text { corpo estranho (sobretudo se houve contacto com solo ou estrume), fraturas expostas, frieiras com ulceração } \\
\text { (frostbite), feridas com necessidade de intervenção cirúrgica não intervencionada nas primeiras seis horas, feridas } \\
\text { em doente com sepsia. }\end{array}$} \\
\hline \multicolumn{5}{|c|}{$\begin{array}{l}{ }^{1} \text { Administrar reforço se última dose há > } 10 \text { anos. Não administrar se há registo de cinco doses administradas. }{ }^{2} \\
{ }^{2} \text { Administrar reforço se última dose há >5 anos. Não administrar se há registo de cinco doses administradas. }{ }^{24} \\
\text { Ponderar dose adicional em caso de feridas de alto risco e última dose há >10 anos. }{ }^{21} \\
{ }^{3} \text { Administrar se ferida muito contaminada ou com muito tecido desvitalizado. }{ }^{21,24} \text { Ponderar administração em } \\
\text { imunodeprimidos ou utilizadores de drogas por via endovenosa. }\end{array}$} \\
\hline
\end{tabular}

tendo em conta que a resposta imunitária associada ao componente acelular da tosse convulsa desaparece rapidamente, seriam necessários reforços mais frequentes e não a cada dez anos ${ }^{6}$ e, por isso, o uso de uma vacina combinada não está indicado.

Pelas razões acima referidas, atualmente a difteria não representa um problema de saúde pública e os programas de vacinação contra a difteria não são considerados prioritários. Contudo, deveriam ser implementadas ações de formação médica contínua no que diz respeito ao diagnóstico e tratamento de doenças alvo de vacinação, pois até $1 / 3$ dos casos de difteria ocorreram em indivíduos que receberam uma ou mais doses. ${ }^{6}$

\section{PREVENÇÃO DO TÉTANO EM CASO DE FERIDAS}

Neste sentido, tendo em conta que nos próximos anos parte da população idosa continuará suscetível ao tétano, torna-se prioritário investir em ações de formação relativamente à abordagem correta das feridas. Enquanto se aguardam testes capazes de determinar a real necessidade de administrar uma nova dose de reforço ou de imunoglobulina específica (e.g., ProTetanus), ${ }^{28}$ continua a ser importante aferir se houve administração dum ciclo completo de primovacinação (três doses), quando foi a última administração e qual o potencial tetanogénico da ferida (Quadro III).

Dados publicados revelam que podemos confiar nos utentes que afirmam ter recebido um reforço nos últimos dez anos; ${ }^{29}$ contudo, a implementação de um sistema de registos, nacional e de fácil consulta em contexto de urgência, seria vantajoso num futuro próximo. Mais, não há qualquer necessidade de administrar um novo ciclo de três doses em caso de primovacinação incompleta, respeitando o lema espanhol dosis puesta, dosis que cuenta. ${ }^{21}$

\section{CONCLUSÃO}

No que diz respeito à vacinação contra o tétano, o novo PNV reduz o número de reforços recomendados na idade adulta, com consequente redução do risco de iatrogenia, diminuição dos custos em saúde, sem aumentar o risco de difteria. Considerando que nas últimas décadas a maioria dos casos de tétano ocorreram na população idosa, é fundamental manter uma postura pró-ativa, promovendo a atualização do esquema vacinal com pelo menos uma vacina após os 65 anos de idade e garantindo a correta abordagem das feridas em contexto de urgência.

\section{AGRADECIMENTOS}

O autor gostaria de agradecer ao Dr. Juan Gérvas pela leitura crítica do manuscrito final.

\section{REFERÊNCIAS BIBLIOGRÁFICAS}

1. World Health Organization. Tetanus vaccine: WHO position paper. Wkly Epidemiol Rec. 2006;81(20):198-208. 
2. Leça A, Calé E, Freitas $G$, Castelão MI, Valente P, Fernandes T. Boletim Vacinação [Internet]. 2012;(5). Lisboa: Direção-Geral da Saúde; 2012 [cited 2016 Aug 11]. Available from: https://www.dgs.pt/documentos-e-publicacoes/boletim-vacinacao-n-5-julho-2012-jpg.aspx

3. Castro L, Gonçalves G, Catarino J. Caracterização epidemiológica dos casos declarados de tétano: oportunidades perdidas de vacinação [Reported cases of tetanus in the North of Portugal (1993-2002): missed opportunities for vaccination]. Acta Med Port. 2004;17(3):225-30. Portuguese

4. Direção-Geral da Saúde. Programa nacional de vacinação 2017 [Internet]. Lisboa: DGS; 2016 [cited 2016Aug 25].Available from: https://www.sns.gov.pt/wpcontent/uploads/2016/06/programa_vacinacao_sns.pdf

5. LaForce FM. Routine tetanus immunizations for adults: once is enough. J Gen Intern Med. 1993;8(8):459-60.

6. Palomo Cobos L. Efectividad de la vacunación antitetánica a los 10 años de la última dosis en una población adulta [Effectiveness of tetanus vaccination in an adult population 10 years after the last dose].Aten Primaria. 1994;14(4):70710. Spanish

7. Gonçalves G, Santos MA, Frade JG, Cunha JS. Levels of diphtheria and tetanus specific IgG of Portuguese adult women, before and after vaccination with adult type Td. Duration of immunity following vaccination. BMC Public Health. 2007;7:109.

8. Balestra DJ, Littenberg B. Should adult tetanus immunization be given as a single vaccination at age 65? A cost-effectiveness analysis. J Gen Intern Med. 1993;8(8):405-12.

9. Geraldes F, Queirós L, Santos MA. Nível elevado de anticorpos anti-tetânicos [Increased level of tetanus antibody twenty one years after a booster dose of tetanus vaccine]. Acta Med Port. 2004;17(2):183-6. Portuguese

10. Gardner P. Issues related to the decennial tetanus-diphtheria toxoid booster recommendations in adults. Infect Dis Clin North Am. 2001;15(1):143-53.

11. Bakshi R, Graves MC. Guillain-Barré syndrome after combined tetanus-diphtheria toxoid vaccination. J Neurol Sci. 1997;147(2):201-2.

12. Baxter R, Bakshi N, Fireman B, Lewis E, Ray P, Vellozzi C, et al. Lack of association of Guillain-Barré syndrome with vaccinations. Clin Infect Dis. 2013;57(2):197-204.

13. Harte CM, Slattum PW. Recommended immunizations for older adults: a primer for pharmacists. Consult Pharm. 2015;30(4):210-20.

14. Goronzy JJ, Weyand CM. Understanding immunosenescence to improve responses to vaccines. Nature Immunol. 2013;14(5):428-36.

15. Weston WM, Friedland LR, Wu X, Howe B. Vaccination of adults 65 years of age and older with tetanus toxoid, reduced diphtheria toxoid and acellular pertussis vaccine (Boostrix ${ }^{\circledR}$ ): results of two randomized trials. Vaccine. 2012;30(9): 1721-8.
16. Pachón delAmo I, Limia SánchezA, Peña-Rey Lorenzo I, Pérez Martín JJ, Rodríguez Recio MJ, García Rodríguez F, et al. Vacunación en adultos - Recomendaciones: vacuna de difteria y tétanos [Internet]. Madrid: Ministerio de Sanidad y Consumo; 2009. Available from: http://www.msssi.gob.es/ciudadanos/proteccionSalud/vacunaciones/docs/TetanosDifteria_2009.pdf

17. Centers for Disease Control and Prevention. Epidemiology and prevention of vaccine-preventable diseases: tetanus [Internet]. Atlanta: CDC; 2012 [updated 2015 Sep 5; cited 2016 Sep 20]. Available from: https://www.cdc.gov/vaccines/pubs/pinkbook/tetanus.html

18. Weinberger B. Adult vaccination against tetanus and diphtheria: the European perspective. Clin Exp Immunol. 2017;187(1):93-9.

19. Department of Health. Tetanus. In: Immunisation against infectious disease: the Green Book. London: Department of Health; 2013. p. 367-84. Available from: https:/www.gov.uk/government/publications/tetanus-the-green-book-chapter-30

20. Palomo Cobos L. Estudio seroepidemiológico de la inmunidad antitetánica posvacunal en Barrado (Cáceres). Rev San Hig Pública. 1987;61(11-12):1201-8.

21. Palomo Cobos L. La efectividad de las vacunaciones sistemáticas en España.Aten Primaria. 1991;(8):503-8.

22. Palomo Cobos L. Estrategias de vacunación antitetánica en el adulto. Bol REAP. 1997;2(2):2. Available from: http://www.reap.es/boletin/BOLETINREAP1997_ ABR_JUN.pdf

23. Trucchi C, Zoppi G. Decennial diphtheria-tetanus adult boosters: are they really necessary? J Prev Med Hyg. 2015;56(1):E44-8.

24. Cooke MW. Are current UK tetanus prophylaxis procedures for wound management optimal? Emerg Med J. 2009;26(12):845-8.

25. Hagen PT, Bond AR, Rehman H, Molella RG, Murad MH. Have you had a tetanus booster in the last 10 years? Sensitivity and specificity of the question. Patient Educ Couns. 2008;70(3):403-6.

\section{CONFLITO DE INTERESSES}

Os autor declara não ter conflitos de interesses.

\section{ENDEREÇO PARA CORRESPONDÊNCIA \\ Fabrizio Cossutta \\ E-mail: fabrizio.cossutta@arslvt.min-saude.pt}

Recebido em 13-11-2016

Aceite para publicação em 19-12-2017

\section{ABSTRACT}

\section{UPDATE OF NATIONAL IMMUNIZATION PROGRAM AND TETANUS VACCINATION}

Tetanus is a severe infectious disease caused by the contamination of wounds by spores of Clostridium tetani. Immunization is the only intervention able to produce immunity, but administration of several boosters is required to maintain protective and long-lasting antibody levels. Recent changes have been made to the Portuguese immunisation scheme, to come into force in 2017. These changes include a reduction in the number of recommended boosters, in line with recommendations from other European Union countries. The aim of this paper is to substantiate and analyse the evidence supporting these changes.

Keywords: Tetanus; Diphtheria-tetanus vaccine; Immunization programs 